\title{
Linear Odd Poisson Bracket on Grassmann Variables
}

\author{
Vyacheslav A. Soroka f \\ Institute of Theoretical Physics \\ National Science Center \\ "Kharkov Institute of Physics and Technology" \\ 310108, Kharkov, Ukraine
}

\begin{abstract}
A linear odd Poisson bracket (antibracket) realized solely in terms of Grassmann variables is suggested. It is revealed that the bracket, which corresponds to a semisimple Lie group, has at once three Grassmann-odd nilpotent $\Delta$-like differential operators of the first, the second and the third orders with respect to Grassmann derivatives, in contrast with the canonical odd Poisson bracket having the only Grassmann-odd nilpotent differential $\Delta$-operator of the second order. It is shown that these $\Delta$-like operators together with a Grassmann-odd nilpotent Casimir function of this bracket form a finite-dimensional Lie superalgebra.
\end{abstract}

PACS: 02.20.Sv; 11.15.-q; 11.30.Pb

Keywords: Poisson bracket; Lie group; Lie superalgebra

*E-mail: vsoroka@kipt.kharkov.ua 
1. Recently a linear degenerate odd Poisson bracket built only of Grassmann variables has been introduced [1]. It was constructed for this bracket, in contrast with the nondegenerate odd bracket having the only Grassmann-odd nilpotent differential $\Delta$-operator of the second order, at once three Grassmann-odd nilpotent $\Delta$-like differential operators of the first, the second and the third orders with respect to Grassmann derivatives. It was also shown that these $\Delta$-like operators together with a Grassmann-odd nilpotent Casimir function of this degenerate odd bracket form a finite-dimensional Lie superalgebra. In the present paper we extend the above-mentioned results to the case of an arbitrary linear odd Poisson bracket, which is also realized solely in terms of the Grassmann variables and corresponds to a semi-simple Lie group.

2. There is a well-known linear even Poisson bracket given in terms of the commuting (Grassmann-even) variables $X_{\alpha}\left(g\left(X_{\alpha}\right)=0\right)$

$$
\left\{X_{\alpha}, X_{\beta}\right\}_{0}=c_{\alpha \beta}^{\gamma} X_{\gamma}, \quad(\alpha, \beta, \gamma=1, \ldots, N)
$$

where $c_{\alpha \beta}^{\gamma}$ are Grassmann-even $\left(g\left(c_{\alpha \beta}{ }^{\gamma}\right)=0\right)$ constants which, because of the main properties of the even Poisson bracket:

$$
\begin{gathered}
\{A, B+C\}_{0}=\{A, B\}_{0}+\{A, C\}_{0}, \\
g\left(\{A, B\}_{0}\right)=g(A)+g(B) \quad(\bmod 2), \\
\{A, B\}_{0}=-(-1)^{g(A) g(B)}\{B, A\}_{0}, \\
\sum_{(A B C)}(-1)^{g(A) g(C)}\left\{A,\{B, C\}_{0}\right\}_{0}=0, \\
\{A, B C\}_{0}=\{A, B\}_{0} C+(-1)^{g(A) g(B)} B\{A, C\}_{0},
\end{gathered}
$$

are antisymmetric in the two lower indices

$$
c_{\alpha \beta}^{\gamma}=-c_{\beta \alpha}^{\gamma}
$$

and obey the conditions

$$
c_{\alpha \lambda}{ }^{\delta} c_{\beta \gamma}{ }^{\lambda}+c_{\beta \lambda}{ }^{\delta} c_{\gamma \alpha}{ }^{\lambda}+c_{\gamma \lambda}{ }^{\delta} c_{\alpha \beta}{ }^{\lambda}=0 .
$$

A sum with the symbol $(A B C)$ in $(5)$ means a summation over cyclic permutations of the quantities $A, B, C$. In relations (2)-(6) $A, B, C$ are functions of the variables $X_{\alpha}$ and $g(A)$ is a Grassmann parity of the quantity $A$. The linear even bracket (1) plays a very important role in the theory of Lie groups, Lie algebras, their representations and applications (see, for example, [2, 3]).

As in the Lie algebra case, we can define a symmetric Cartan-Killing tensor

$$
g_{\alpha \beta}=g_{\beta \alpha}=c_{\alpha \gamma}{ }^{\lambda} c_{\beta \lambda}{ }^{\gamma}
$$

and verify with the use of relations (8) an anti-symmetry property of a tensor

$$
c_{\alpha \beta \gamma}=c_{\alpha \beta}{ }^{\delta} g_{\delta \gamma}=-c_{\alpha \gamma \beta} .
$$

By assuming that the Cartan-Killing metric tensor is non-degenerate $\operatorname{det}\left(g_{\alpha \beta}\right) \neq 0$ (this case corresponds to the semi-simple Lie group), we can define an inverse tensor $g^{\alpha \beta}$

$$
g^{\alpha \beta} g_{\beta \gamma}=\delta_{\gamma}^{\alpha}
$$


with the help of which we are able to build a quantity

$$
C=X_{\alpha} X_{\beta} g^{\alpha \beta}
$$

that, in consequence of relation (10), is a Casimir function for the bracket (1)

$$
\{C, \ldots\}_{0}=0
$$

3. Now let us replace in expression (1) the commuting variables $X_{\alpha}$ by Grassmann variables $\theta_{\alpha}\left(g\left(\theta_{\alpha}\right)=1\right)$. Then we obtain a binary composition

$$
\left\{\theta_{\alpha}, \theta_{\beta}\right\}_{1}=c_{\alpha \beta}^{\gamma} \theta_{\gamma}
$$

which, due to relations (7) and (8), meets all the properties of the odd Poisson brackets:

$$
\begin{gathered}
\{A, B+C\}_{1}=\{A, B\}_{1}+\{A, C\}_{1}, \\
g\left(\{A, B\}_{1}\right)=g(A)+g(B)+1 \quad(\bmod 2), \\
\{A, B\}_{1}=-(-1)^{(g(A)+1)(g(B)+1)}\{B, A\}_{1}, \\
\sum_{(A B C)}(-1)^{(g(A)+1)(g(C)+1)}\left\{A,\{B, C\}_{1}\right\}_{1}=0, \\
\{A, B C\}_{1}=\{A, B\}_{1} C+(-1)^{(g(A)+1) g(B)} B\{A, C\}_{1} .
\end{gathered}
$$

It is surprising enough that the odd bracket can be defined solely in terms of the Grassmann variables as well as an even Martin bracket [4].

By the way, let us note that we can also construct only on the Grassmann variables a non-linear odd Poisson bracket of the form

$$
\left\{\theta_{\alpha}, \theta_{\beta}\right\}_{1}=c_{\alpha \beta}{ }^{\gamma \delta \lambda} \theta_{\gamma} \theta_{\delta} \theta_{\lambda}
$$

In order to satisfy the property (15), the constants $c_{\alpha \beta}{ }^{\gamma \delta \lambda}$ have to be antisymmetric in the below indices

$$
c_{\alpha \beta}^{\gamma \delta \lambda}=-c_{\beta \alpha}^{\gamma \delta \lambda}
$$

and, for the validity of the Jacobi identities (16), they must obey the following conditions

$$
\sum_{(\alpha \beta \gamma)} c_{\alpha \beta}{ }^{\lambda\left[\delta_{1} \delta_{2}\right.} c_{\lambda \gamma}{ }^{\left.\delta_{3} \delta_{4} \delta_{5}\right]}=0
$$

where square brackets [...] mean the antisymmetrization of the indices in them.

Returning to the linear odd bracket (12) notice, that on functions $A, B$ of Grassmann variables $\theta_{\alpha}$ it has the form

$$
\{A, B\}_{1}=A \overleftarrow{\partial}_{\theta_{\alpha}} c_{\alpha \beta}^{\gamma} \theta_{\gamma} \vec{\partial}_{\theta_{\beta}} B
$$

where $\overleftarrow{\partial}$ and $\vec{\partial}$ are the right and left derivatives and $\partial_{x^{A}} \equiv \frac{\partial}{\partial x^{A}}$. The bracket (12) can be either degenerate or non-degenerate in the dependence on whether the matrix $c_{\alpha \beta}{ }^{\gamma} \theta_{\gamma}$ in the indices $\alpha, \beta$ is degenerate or not. The indices $\alpha, \beta$ can be raised and lowered by means of the non-degenerate metric tensors (9), (11)

$$
\theta^{\alpha}=g^{\alpha \beta} \theta_{\beta}, \quad \partial_{\theta^{\alpha}}=g_{\alpha \beta} \partial_{\theta_{\beta}} .
$$


Hereafter only the non-degenerate metric tensors (11) will be considered.

4. By contracting the indices in a product of the three Grassmann variables with the upper indices $\theta^{\alpha} \theta^{\beta} \theta^{\gamma}$ and of the three successive Grassmann derivatives $\partial_{\theta_{\alpha}} \partial_{\theta_{\beta}} \partial_{\theta_{\gamma}}$, respectively, with the lower indices $\alpha, \beta, \gamma$ in (8), we obtain the relations

$$
\begin{gathered}
\theta^{\alpha} \theta^{\beta} \theta^{\gamma} c_{\alpha \beta}{ }^{\lambda} c_{\lambda \gamma}{ }^{\delta}=0, \\
c_{\alpha \beta}{ }^{\lambda} c_{\lambda \gamma}{ }^{\delta} \partial_{\theta_{\alpha}} \partial_{\theta_{\beta}} \partial_{\theta_{\gamma}}=0,
\end{gathered}
$$

which will be used later on many times. In particular, taking into account relation (18), we can verify that the linear odd bracket (12) has the following Grassmann-odd nilpotent Casimir function

$$
\Delta_{+3}=\frac{1}{\sqrt{3 !}} \theta^{\alpha} \theta^{\beta} \theta^{\gamma} c_{\alpha \beta \gamma}, \quad\left\{\Delta_{+3}, \ldots\right\}_{1}=0, \quad\left(\Delta_{+3}\right)^{2}=0 .
$$

Such a notation for this Casimir function will be clear below.

It is a well-known fact that, in contrast with the even Poisson bracket, the nondegenerate odd Poisson bracket has one Grassmann-odd nilpotent differential $\Delta$-operator of the second order, in terms of which the main equation has been formulated in the Batalin-Vilkovisky scheme [5, 6, 0, 8, 9, 10] for the quantization of gauge theories in the Lagrangian approach. In a formulation of Hamiltonian dynamics by means of the odd Poisson bracket with the help of a Grassmann-odd Hamiltonian $\bar{H}(g(\bar{H})=1)$ 11, 12, 13, 14, 15, 16, 17, 18, 19, 20, 21, 22 this $\Delta$-operator plays also a very important role being used to distinguish the non-dissipative dynamical systems, for which $\Delta \bar{H}=0$, from the dissipative ones [1], for which the Grassmann-odd Hamiltonian satisfies the condition $\Delta \bar{H} \neq 0$.

Now let us try to build the $\Delta$-operator for the linear odd bracket (12). It is remarkable that, in contrast with the canonical odd Poisson bracket having the only $\Delta$-operator of the second order, we are able to construct at once three $\Delta$-like Grassmann-odd nilpotent operators which are differential operators of the first, the second and the third orders respectively

$$
\begin{array}{rlrl}
\Delta_{+1} & =\frac{1}{\sqrt{2}} \theta^{\alpha} \theta^{\beta} c_{\alpha \beta \gamma} \partial_{\theta_{\gamma}}, & \left(\Delta_{+1}\right)^{2}=0 ; \\
\Delta_{-1}=\frac{1}{\sqrt{2}} \theta_{\gamma} c_{\alpha \beta}{ }^{\gamma} \partial_{\theta_{\alpha}} \partial_{\theta_{\beta}}, & \left(\Delta_{-1}\right)^{2}=0 ; \\
\Delta_{-3}=\frac{1}{\sqrt{3 !}} c_{\alpha \beta \gamma} \partial_{\theta_{\alpha}} \partial_{\theta_{\beta}} \partial_{\theta_{\gamma}}, & \left(\Delta_{-3}\right)^{2}=0 .
\end{array}
$$

The nilpotency of the operators $\Delta_{+1}$ and $\Delta_{-1}$ is a consequence of relations (18) and (19).

It is also interesting to reveal that these $\Delta$-like operators together with the Casimir function $\Delta_{+3}(9)$ are closed into the finite-dimensional Lie superalgebra, in which the anticommuting relations between the quantities $\Delta_{\lambda}(\lambda=-3,-1,+1,+3)(20)-(23)$ with the nonzero right-hand side ar£

$$
\left\{\Delta_{-1}, \Delta_{+1}\right\}=Z,
$$

\footnotetext{
${ }^{1}$ In order to avoid a misunderstanding, let us note that below $[A, B]=A B-B A$ and $\{A, B\}=$ $A B+B A$.
} 


$$
\left\{\Delta_{-3}, \Delta_{+3}\right\}=N-3 Z,
$$

where

$$
N=-c^{\alpha \beta \gamma} c_{\alpha \beta \gamma}
$$

is a number of values for the indices $\alpha, \beta, \gamma(\alpha, \beta, \gamma=1, \ldots, N)$ and

$$
Z=D-K
$$

is a center of this superalgebra

$$
\left[Z, \Delta_{\lambda}\right]=0, \quad(\lambda=-3,-1,+1,+3) .
$$

In $(26)$

$$
D=\theta_{\alpha} \partial_{\theta_{\alpha}}
$$

is a "dilatation" operator for the Grassmann variables $\theta_{\alpha}$ which distinguishes the $\Delta_{\lambda^{-}}$ operators with respect to their uniformity degrees in $\theta$

$$
\left[D, \Delta_{\lambda}\right]=\lambda \Delta_{\lambda}, \quad(\lambda=-3,-1,+1,+3),
$$

and the quantity $K$ has the form

$$
K=\frac{1}{2} \theta^{\alpha} \theta^{\beta} c_{\alpha \beta}{ }^{\lambda} c_{\lambda \gamma \delta} \partial_{\theta_{\gamma}} \partial_{\theta_{\delta}} .
$$

The operator $Z$ is also a center of the Lie superalgebra which contains both the operators $\Delta_{\lambda}(20)-(23), Z(26)$ and the operator $D(28)$

$$
[Z, D]=0 .
$$

We can add to this superalgebra the generators

$$
S_{\alpha}=\theta_{\gamma} c_{\alpha \beta}^{\gamma} \partial_{\theta_{\beta}}
$$

with the following commutation relations:

$$
\begin{gathered}
{\left[S_{\alpha}, S_{\beta}\right]=c_{\alpha \beta}{ }^{\gamma} S_{\gamma}, \quad\left[S_{\alpha}, \Delta_{\lambda}\right]=0,} \\
{\left[S_{\alpha}, Z\right]=0, \quad\left[S_{\alpha}, D\right]=0 .}
\end{gathered}
$$

In order to prove the permutation relations for the Lie superalgebra (20)-(33), we have to use relations (18) and (19). Note that the center $Z$ (26) coincides with the expression for a quadratic Casimir operator of the Lie algebra (33a) for the generators $S_{\alpha}$ given in the representation (32)

$$
S_{\alpha} S_{\beta} g^{\alpha \beta}=Z .
$$

5. Thus, we see that both the even and odd linear Poisson brackets are internally inherent in the Lie group with the structure constants subjected to conditions (7) and (8). However, only for the linear odd Poisson bracket realized in terms of the Grassmann variables and only in the case when this bracket corresponds to the semi-simple Lie group, there exists the Lie superalgebra (20)-(33) for the $\Delta$-like operators of this bracket. 
Note that in the case of the degenerate Cartan-Killing metric tensor (9), relation (10) remains valid and we can construct only two $\Delta$-like Grassmann-odd nilpotent operators: $\Delta_{-1}(22)$ and $\Delta_{-3}(23)$, which satisfy the trivial anticommuting relation

$$
\left\{\Delta_{-1}, \Delta_{-3}\right\}=0
$$

The Lie superalgebra (20)-(33), naturally connected with the linear odd Poisson bracket (12), may be useful for the subsequent development of the Batalin-Vilkovisky formalism for the quantization of gauge theories. Indeed, very similar to (12) odd Poisson brackets on the Grassmann algebra are used in a generalization [23]] of the triplectic formalism [24] which is a covariant version of the $S p(2)$-symmetric quantization [7] of general gauge theories. We should therefore expect that the Lie superalgebra (20)-(33), closely related with the linear odd bracket (12), will also find the application for the further development of the above-mentioned generalization of the triplectic formalism. Let us note that the superalgebra (20)-(33) can also be used in the theory of representations of the semi-simple Lie groups.

The author is sincerely thankful to V.D. Gershun for useful discussions.

This work was supported, in part, by the Ukrainian State Foundation of Fundamental Researches, Grant No 2.5.1/54, by Grant INTAS No 93-127 (Extension) and by Grant INTAS No 93-633 (Extension).

\footnotetext{
${ }^{2}$ This paper appeared after the present work had been finished.
} 


\section{References}

[1] V.A. Soroka, Degenerate odd Poisson bracket on Grassmann variables, hepth/9811223.

[2] F.A. Berezin, Introduction to algebra and analysis with anticommuting variables, (Moscow State University, 1983).

[3] M.V. Karasev, V.P. Maslov, Non-linear Poisson brackets. Geometry and quantization, (Moscow, Nauka, 1991).

[4] J.L. Martin, Proc. Roy. Soc. A 251 (1959) 536.

[5] I.A. Batalin, G.A. Vilkovisky, Phys. Lett. B 102 (1981) 27.

[6] I.A. Batalin, G.A. Vilkovisky, Phys. Rev. D 28 (1983) 2567.

[7] I.A. Batalin, P.M. Lavrov, I.V. Tyutin, J. Math. Phys. 31 (1990) 1487.

[8] I.A. Batalin, I.V. Tyutin, Int. J. Mod. Phys. A 8 (1993) 2333.

[9] A.S. Schwarz, Commun. Math. Phys. 155 (1993) 249.

[10] O.M. Khudaverdian, A.P. Nersessian, Mod. Phys. Lett. A 8 (1993) 2377.

[11] D.A. Leites, Docl. Acad. Nauk SSSR 236 (1977) 804.

[12] D.V. Volkov, A.I. Pashnev, V.A. Soroka and V.I. Tkach, JETP Lett. 44 (1986) 70; Teor. Mat. Fiz. 79 (1989) 117.

[13] V.A. Soroka, Lett. Math. Phys. 17 (1989) 201.

[14] O.M. Khudaverdian, J. Math. Phys. 32 (1991) 1934.

[15] O.M. Khudaverdian, A.P. Nersessian, J. Math. Phys. 32 (1991) 1938.

[16] D.V. Volkov, V.A. Soroka, V.I. Tkach, Yad. Fiz. 44 (1986) 810.

[17] D.V. Volkov, V.A. Soroka, Yad. Fiz. 46 (1987) 110.

[18] V.A. Soroka, JETP Lett. 59 (1994) 219.

[19] V.A. Soroka, Odd Poisson bracket in Hamilton's dynamics, in: Proceedings of the Workshop on Variational and Local Methods in the Study of Hamiltonian Systems, (ICTP, Trieste, Italy, 24-28 October, 1994), eds. A. Ambrosetti and G.F. Dell'Antonio (World Scientific, Singapore, 1995) p. 192; hep-th/9503214.

[20] A.P. Nersessian, JETP Lett. 58 (1993) 64.

[21] D.V. Volkov, A.V. Tur , V.V. Yanovsky, Phys. Lett. A 203 (1995) 357.

[22] V.A. Soroka, Phys. Atom. Nucl. 59 (1996) 1270; hep-th/9507030.

[23] M.A. Grigoriev, A Lie group structure underlying the triplectic geometry, hepth/9901046.

[24] I.A. Batalin, R. Marnelius and A.M. Semikhatov, Nucl. Phys. B 446 (1995) 249. 